\title{
The Adenovirus Type 3 Dodecahedron's RGD Loop Comprises an HSPG Binding Site That Influences Integrin Binding
}

\author{
E. Gout, ${ }^{1,2,3}$ G. Schoehn, ${ }^{1,2,3,4}$ D. Fenel, ${ }^{1,2,3}$ H. Lortat-Jacob, ${ }^{1,2,3}$ and P. Fender ${ }^{1,2,3,4}$ \\ ${ }^{1}$ CNRS-Institut de Biologie Structurale, 41 rue Jules Horowitz, 38027 Grenoble, France \\ ${ }^{2}$ CEA-Institut de Biologie Structurale, 41 rue Jules Horowitz, 38027 Grenoble, France \\ ${ }^{3}$ UJF-Institut de Biologie Structurale, 41 rue Jules Horowitz, 38027 Grenoble, France \\ ${ }^{4}$ Unit for Virus Host Cell Interaction UMI 3265, 6 rue Jules Horowitz, 38042 Grenoble Cedex 09, France \\ Correspondence should be addressed to P. Fender, pfender@embl.fr
}

Received 1 October 2009; Accepted 17 December 2009

Academic Editor: Gerald Schumann

Copyright (๑) 2010 E. Gout et al. This is an open access article distributed under the Creative Commons Attribution License, which permits unrestricted use, distribution, and reproduction in any medium, provided the original work is properly cited.

Human type 3 adenovirus dodecahedron (a virus like particle made of twelve penton bases) features the ability to enter cells through Heparan Sulphate Proteoglycans (HSPGs) and integrins interaction and is used as a versatile vector to deliver DNA or proteins. Cryo-EM reconstruction of the pseudoviral particle with Heparan Sulphate (HS) oligosaccharide shows an extradensity on the RGD loop. A set of mutants was designed to study the respective roles of the RGD sequence (RGE mutant) and of a basic sequence located just downstream. Results showed that the RGE mutant binding to the HS deficient CHO-2241 cells was abolished and unexpectedly, mutation of the basic sequence (KQKR to AQAS) dramatically decreased integrin recognition by the viral pseudoparticle. This basic sequence is thus involved in integrin docking, showing a close interplay between HSPGs and integrin receptors.

\section{Introduction}

Human adenoviruses (Ads) are nonenveloped viruses responsible for respiratory, ocular, and enteric infections. Their icosahedral capsid, containing the $36-\mathrm{kpb}$ double-stranded DNA genome, is composed of three major proteins: the hexon, the penton base, and the fibre. At the 12 vertices of the capsid, the protruding fiber is noncovalently attached to the penton base. It has been reported that the fibre interacts with high affinity with a primary receptor, enabling a subsequent interaction of the penton base RGD motif to cellular integrins that trigger endocytosis $[1,2]$. Remarkably, HAd3 penton-base expressed in the baculovirus system led to the formation of symmetric complex of 12 pentameric penton bases called base-dodecahedron (Bs-Dd) [3]. A similar particle harbouring fibres is naturally produced during the HAd3 replication cycle. We have previously reported that Bs-Dd interacts with cellular Heparan Sulfate Proteoglycans (HSPGs) and facilitates in turn the particle binding to integrins that is a prerequisite for entry $[4,5]$. Due to its great internalisation efficiency, dodecahedron has already been exploited as a versatile vector in DNA or protein delivery $[3,6,7]$. Here, we describe that a Heparan Sulphate (HS) oligosaccharide binds to the Bs-Dd «RGD-loop» and that a basic sequence located next to the RGD motif is critical for integrin recognition.

\section{Materials and Methods}

2.1. HS Oligosaccharide. HS octasaccharide (dp8) was obtained by partial heparinase I digestion of heparin and purification by size exclusion chromatography. This dp8 oligosaccharide is made of four sulfated dissacharide repeats of N-sulfated glucosamine and iduronic acid. For cryomicroscopy reconstruction, the structure of an octamer of $\mathrm{N}$-acetyl glucosamine (PDB 1EHN) has been used due to its similarity to dp8.

2.2. CryoEM. Bs-Dd $(0,5 \mathrm{mg} / \mathrm{mL})$ was incubated for $18 \mathrm{H}$ at $4^{\circ} \mathrm{C}$ with $\mathrm{dp} 8(0,5 \mathrm{mg} / \mathrm{mL})$ in PBS, corresponding to a 20: 1 ratio of oligosaccharide per penton base monomer. Excess of dp8 was withdrawn by 8 cycles of centrifugation 
and PBS addition in a microconcentator (microcon 30,000 MWCO; Millipore). The sample was prepared for cryo-EM observation as described before [8]. 1,200 single Bs-Dd/dp8 particles out of a total of 2,000 coming from 16 micrographs imaged with a JEOL 2010 FEG electron microscope at a magnification of 50,000 times were used to generate the $3 \mathrm{D}$ structure of the complex. The resolution of the 3D structure of this virus was estimated to be $20 \AA$ resolution $(0.5$ cutoff for the Fourier Shell Correlation).

2.3. Mutations in the RGD Loop. Mutations were achieved using the Stratagene «Quick change mutation kit» on the pAcUW31 vector encoding the wild type HAd3 penton base. After DNA sequencing to control mutation, baculovirus were built according to the Clontech protocol, as previously described [3]. Protein expression was detected by western blotting, using "home-made" rabbit anti-Ad3 penton base antibody and mutants were then purified by ultracentrifugation onto a $15-40 \%$ sucrose gradient, as previously described [3]. Mutated dodecahedra were recovered in the bottom fractions and dialysed against PBS. Negative staining electron microscopy was performed using uranyl acetate staining and grids were observed on Philips CM10 electron microscope.

2.4. FACS Analysis. HeLa or $\mathrm{CHO}$ cells were resuspended in PBS $\left(5.10^{5}\right.$ cells in $\left.200 \mu \mathrm{l}\right)$ supplemented or not with $1 \mathrm{mM} \mathrm{CaCl}_{2}$. Cells were incubated for $1 \mathrm{H}$ at $4^{\circ} \mathrm{C}$ with equal amounts $(15 \mathrm{ug} / \mathrm{mL})$ (micrograms) of either Bs-Dd, MutAQAS, or MutRGE. After PBS washes, cells were incubated with a "home-made" rabbit serum directed against the Ad3 penton base, then with FITC labelled secondary antibody. Cells were analysed by Flow Cytometry using the FACS fluorescein channel (Becton Dickinson; CellQuest). For experiments using the integrin blocking drug, cells were preincubated with $3 \mathrm{nM}$ of S36578 (Kindly given by Dr Tucker, Servier Laboratory) for 30 minutes at $4^{\circ} \mathrm{C}$ prior to dodecahedron addition. For experiments using oligosaccharide, dp8 $(10 \mu \mathrm{g} / \mathrm{mL})$ was preincubated with dodecahedron for 30 minutes at $4{ }^{\circ} \mathrm{C}$ prior to the incubation with cells.

\section{Results and Discussion}

3.1. Cryo-EM Reconstruction of Bs-Dd/Octasaccharide Complex. In order to localize the HSPG recognition surface on the Bs-Dd particle, cryo-electron microscopy (cryo-EM) was performed on Bs-Dd alone or preincubated with an HS octasaccharide (dp8). Reconstruction of Bs-Dd/dp8 particles was performed to generate the $3 \mathrm{D}$ structure of the complex at a resolution estimated to $20 \AA$ (Figure 1(a)). An extradensity located on spikes at the top of the particle corresponding to the RGD loop [8-10] was clearly visible in the reconstruction of Bs-Dd/dp8 complex when compared to the previously determined Bs-Dd structure filtered to $20 \AA$ [8] (Figure 1(a)). As shown in Figure 1(b), this trilobed density can easily accommodates an octamer of $\mathrm{N}$-acetyl glucosamine (which should basically have the same length as our dp 8 molecule and of a similar structure). The extra density has the shape of an isosceles triangle with one less defined edge (marked
"\#” on Figure 1(b)) suggesting that only the right part of the oligosaccharide is attached to the Bs-Dd RGD loop; the left part is floating around and occupying a different position on the "\#” marked edge in Figure 1(b). Such location for an HSPG binding site suggests a close relationship between HSPG and integrin recognition that are both involved in BsDd attachment and entry into the cell $[4,5]$. This observation is reinforced by the presence of a consensus $(+\mathrm{x}++)$ basic sequence known to interact with HS [11] just downstream the RGD motif (Figure 2(a)).

3.2. Mutations in the RGD Loop of $B s-D d$. In order to investigate the role played by this basic sequence, mutations were performed to change KQKR into AQAS (MutAQAS) and an RGD-to-RGE mutation (MutRGE) was also produced to investigate the role played by integrins (Figure 2(a)). Baculovirus-expressed mutants were checked by westernblotting using an anti-dodecahedron serum (Figure 2(b)) and then purified by ultracentrifugation on sucrose gradient. Negative staining electron microscopy showed that neither pentamerisation nor dodecamerisation of the proteins was affected by the mutations (Figure 2(b)).

3.3. Binding of Mutants on HSPG Expressing HeLa Cells with or without S36578. We have previously demonstrated that HSPGs are the main receptors involved in Bs-Dd attachment to HeLa cells. Neutralisation of the putative HS binding site in the particle would then result in a decrease of its binding to this cell line. To investigate this point, HeLa cells were incubated at $4^{\circ} \mathrm{C}$ with equal amounts of either BsDd, MutAQAS or MutRGE. Bound particles were detected with appropriated antibodies and cells were analysed by Flow Cytometry. As expected, a strong binding of Bs-Dd was observed, contrasting with the low background raised by the control cells incubated with antibodies only (Figure 3(a), green and black histograms). A weak but relevant shift of the MutRGE signal towards the lower intensity was observed (purple histogram), showing that direct attachment to integrins was prevented, but that the main attachment to HSPGs still occurred. Interestingly, binding of MutAQAS (light blue histogram) was dramatically reduced, suggesting a significant contribution of the KQKR sequence to cellular receptor recognition likely through reduction of HSPG recognition.

To better understand the role played by integrins in the attachment, similar experiments were performed on cells preincubated for 30 minutes at $4^{\circ} \mathrm{C}$ with the integrin blocking drug S36578 as previously described [5]. A similar profile was observed (Figure 3(a), right panel). To look closer to the effect of $\$ 36578$ addition on particles binding, each mutant was individually compared in respect of the presence or absence of the integrin blocking drug (Figure 3(a), bottom panels). It was clear that integrin inhibition by the drug slightly decreased the wild type particle binding, reflecting the contribution of integrins direct attachment occurring beside the main attachment to HSPGs. A similar trend was seen with MutRGE in presence of the S36578 drug, showing that the RGD-to-RGE mutation was not sufficient to totally 

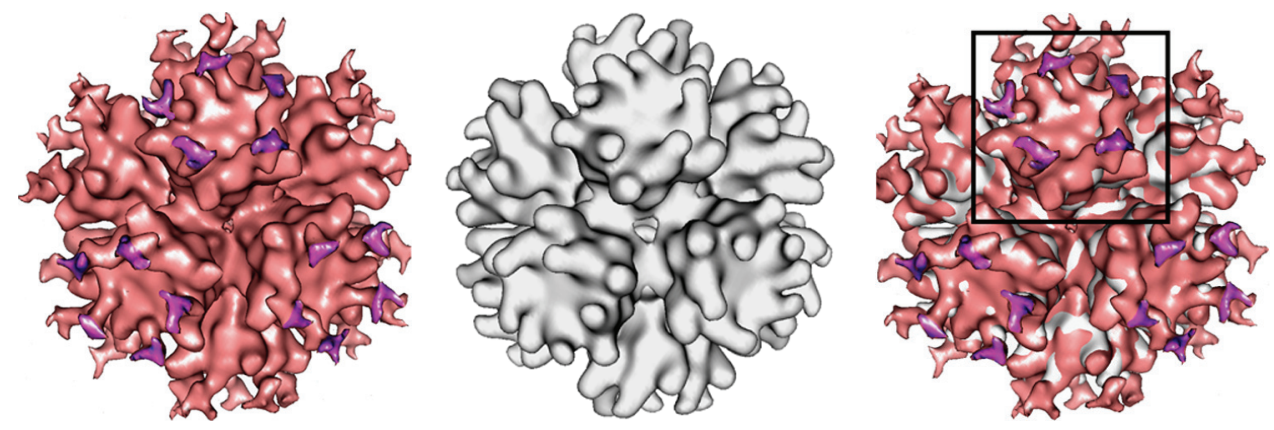

$5 \mathrm{~nm}$

(a)
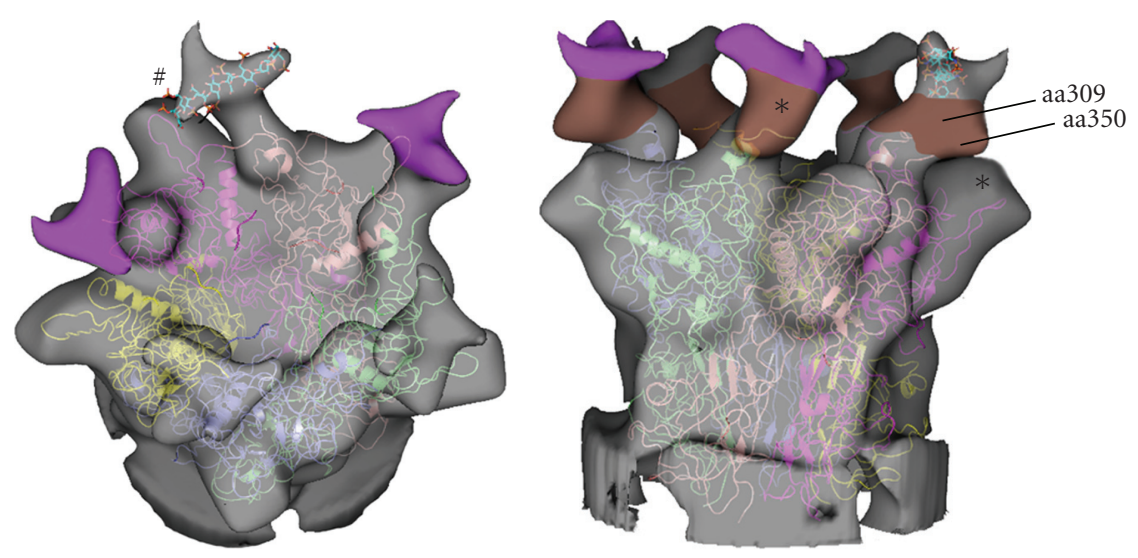

$3 \mathrm{~nm}$

(b)

Figure 1: Cryo-EM reconstruction of Bs-Dd in complex to an HS oligosaccharide. (a) Bs-Dd was incubated with dp8 and the cryo-EM reconstruction of the complex (left panel; pink and purple) was compared to the Bs-Dd alone (middle panel; gray) by sur-imposing them (right panel). The purple density is attributed to the dp8 presence, the pink one to the Bs-Dd. (b) Fitting of both the HAd2 penton base atomic structure (PDB 1X9P) and of an octamer of N-acetyl glucosamine (PDB 1EHN) represented in blue into a Bs-Dd pentamer extradensity viewed in a top view (left) and side view (right) orientation. The symbol "\#” highlights the less defined edge of the extra density suggesting therefore that the molecule occupying this part is more flexible compared to the opposite vertex. Asterisks denote the missing part of the RGD loop in the HAd2 X-ray structure (brown part) as well as the hyper variable loop (grey part) compared to the Bs-Dd structure.

abolish the direct integrin recognition on HeLa cells. On the contrary, MutAQAS binding was not affected by the S36578 drug treatment. This was unexpected as it was thought, in light of Figure 1, that this sequence was only involved in HSPG but not in integrin recognition. This nonresponse to the S36578 drug suggests that beside a reduction in HSPG recognition on HeLa cells, integrin binding is abolished by this mutation despite the presence of an intact RGD sequence.

\subsection{Binding of Mutants on CHO-2241 HSPG Deficient Cells} with or without S36578. To further investigate the role of the basic sequence on integrin binding, a similar experiment was performed using HS deficient CHO-2241 cells. Bs-Dd binding to this cell line was observed as seen on Figure 3(b) (left panel, blue histogram). On these cells, integrins are the unique Bs-Dd receptor, as pretreatment with S36578 drug totally abolished particle binding (Figure 3(b), right panel). Interestingly, MutRGE was not able to bind these cells (even in absence of S36578) indicating that mutation as discrete as conversion of an aspartate to glutamate (only one supplementary methyl in the lateral chain) was sufficient to totally prevent dodecahedron docking and thus that integrins are the unique receptor for Bs-Dd on this cell line. Surprisingly, MutAQAS supposed to be affected only in HSPG recognition showed a reduced binding capacity on this HSPG deficient cell line $(80 \%$ of inhibition in absence of S3578, Figure 3(b) left panel) compared to Bs-Dd. This experiment reinforces the previous observation on HeLa cell showing that despite the presence of an intact RGD sequence, mutation in the KQKR sequence affects integrin recognition. This observation is in agreement with the hypothesis stating that although eight different integrins have been shown to utilise the RGD sequence, their specificity might be principally governed by the local conformation adopted by this tripeptide in a particular ligands [12]. As Adenovirus penton base displaying a great variation in the RGD loop size (42 aminoacids for HAd3 but only 14 for HAd12 and up to 75 


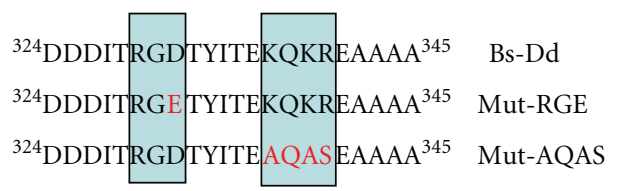

(a)

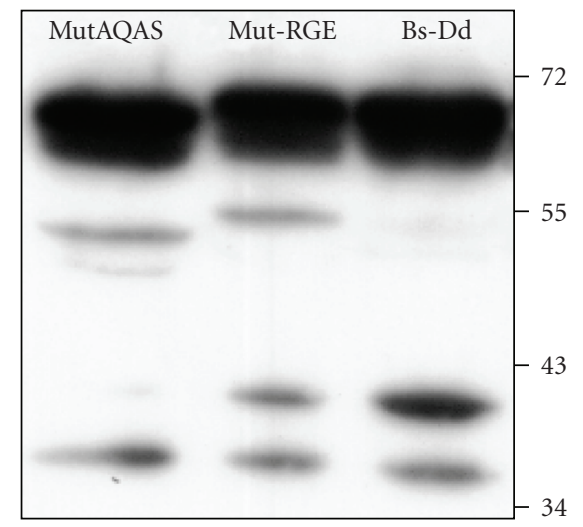

(b)
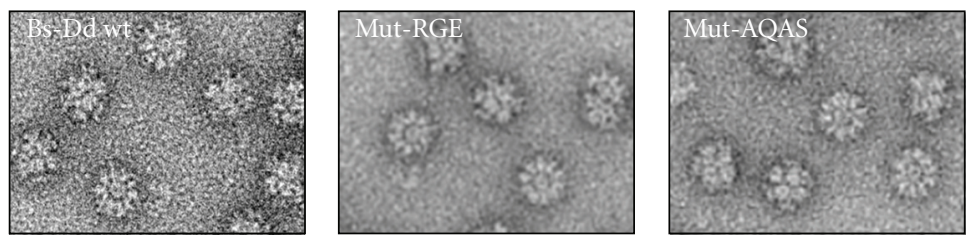

(c)

FIGURe 2: Mutations in the Bs-Dd RGD loop. (a) Sequence of Bs-Dd mutants in either the RGD or the basic motif (blue boxes). Mutations are indicated in red. (b) Detection of baculovirus expressed protein by western-blot using an anti-dodecahedron serum. (c) Negative staining micrographs of purified mutants and wild type Bs-Dd showing that dodecamerisation is not affected by the mutations.

for HAd2), it could be conceivable that integrin recognition is influenced by the loop flexibility. Beside the length of the loop, its aminoacids composition may also play an important role. Indeed, it has been reported for the HAd2 penton base that replacement of the RGD flanking region (HAIRGDTFA to SFGRGDIRN) did not impair $\alpha \mathrm{v} \beta 3$ recognition, but nearly abolished $\alpha \mathrm{v} \beta 5$ and $\alpha 5 \beta 1$ binding $[12,13]$. By mimicking the fibronectin sequence (VTGRGDSPA), Ad2 derived penton base exhibited high binding to $\alpha \mathrm{v} \beta 3$ and $\alpha 5 \beta 1$ but not to $\alpha \mathrm{v} \beta 5$. In the light of our results, it is now clear that the putative basic HSPG binding site next to the RGD sequence is implicated in the recognition of integrins.

\subsection{HS Octasaccharide Partially Restores Bs-Dd Binding to} Integrin in the Absence of Calcium. To test whether HS binding to Bs-Dd influences the integrin recognition efficiency, CHO-2241 cells were incubated in PBS $1 \mathrm{mM} \mathrm{CaCl}_{2}$ at $4^{\circ} \mathrm{C}$ with different concentrations of $\mathrm{Bs}-\mathrm{Dd}$, with or without $10 \mu \mathrm{g} / \mathrm{ml}$ of $\mathrm{dp} 8$ (Figures $4(\mathrm{a})$ and $4(\mathrm{~b})$ ). Particle binding was detected by Flow Cytometry. Histograms clearly showed that oligosaccharide had no effect on binding whatever the Bs-Dd concentration. On the contrary, a similar experiment performed in PBS without calcium showed a different feature (Figure 4(a) and 4(b)). Indeed, Bs-Dd binding to CHO-2241 was completely abolished in the absence of calcium indicating that cations were required for interaction. Surprisingly, Bs-Dd binding to $\mathrm{CHO}-2241$ was partially restored upon dp8 addition. This effect could be explained by a structural change induced by the oligosaccharide on the RGD loop resulting in a change of the affinity for integrins. Indeed, it is known that the RGD loop in the adenoviruses is flexible and normally not resolved by cryo-EM and image analysis neither in the HAd5 cryo-EM structure [14] nor in the HAd3 Bs-Dd cryo-EM structure (Fuschiotti et al., [8]). The fact that this loop is longer in complex with oligosaccharide compared to the Bs-Dd alone (Figure 1(a) and brown part in Figure 1(b) right panel) could be explained by its rigidification induced by a structural change (and enabled us to visualize the $\operatorname{dp} 8$ at the apical end of this protuberance). This rigidification has no effect on binding under favourable conditions (i.e., in presence of cations, Figure 4) but seems crucial when interaction between the particle and the receptor is weakened by the absence of the stabilizing cation $[15,16]$. It could be also hypothesized that Bs-Dd, integrins, and HS would be associated in a ternary complex in which the HS oligosaccharide could consolidate the interaction by forming a bridge between the pseudoviral particle and integrins. Indeed, close relationship between integrins and HSPGs has been described for different ligands such as extracellular matrix proteins like fibronectin, but 

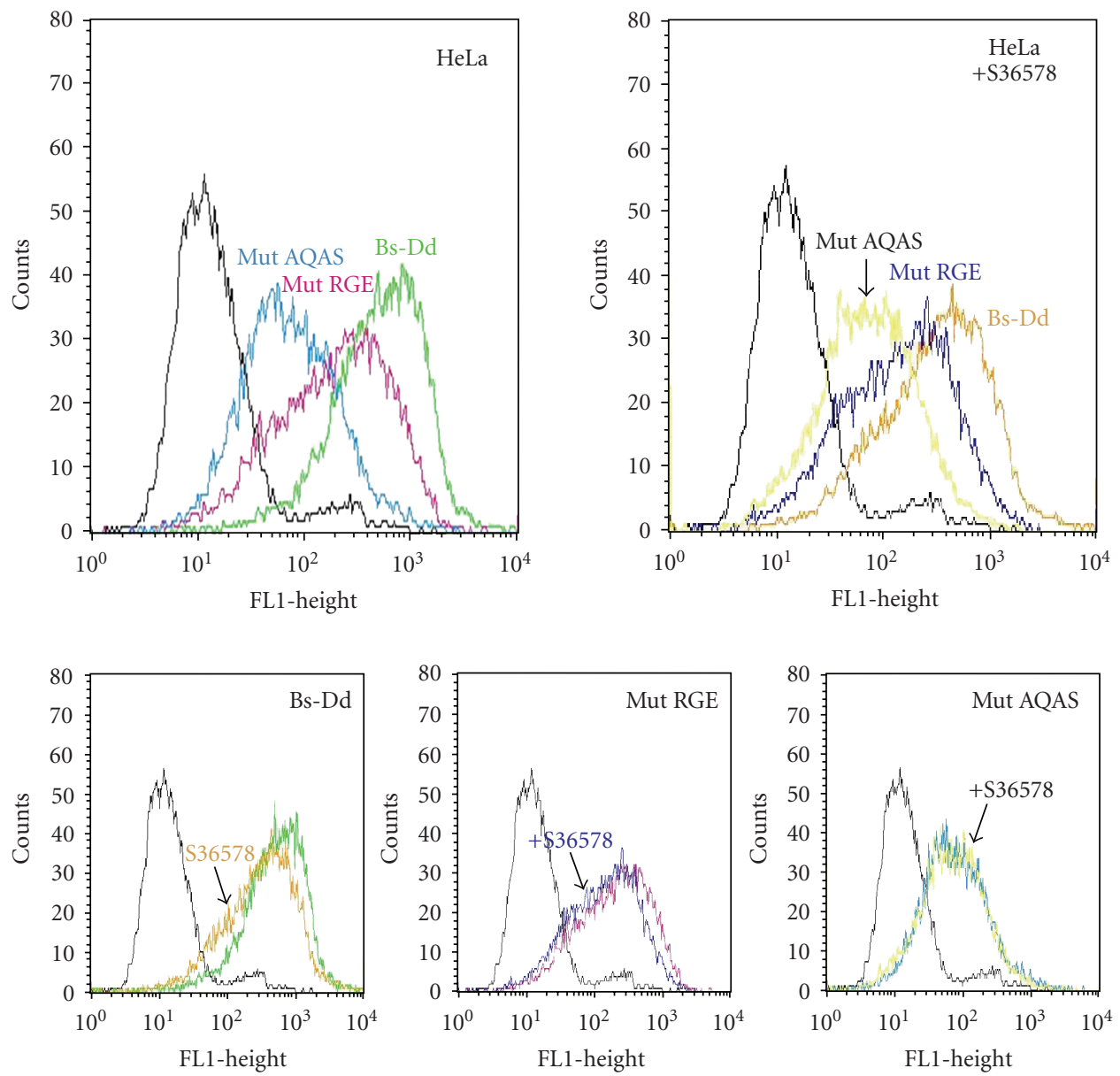

(a)
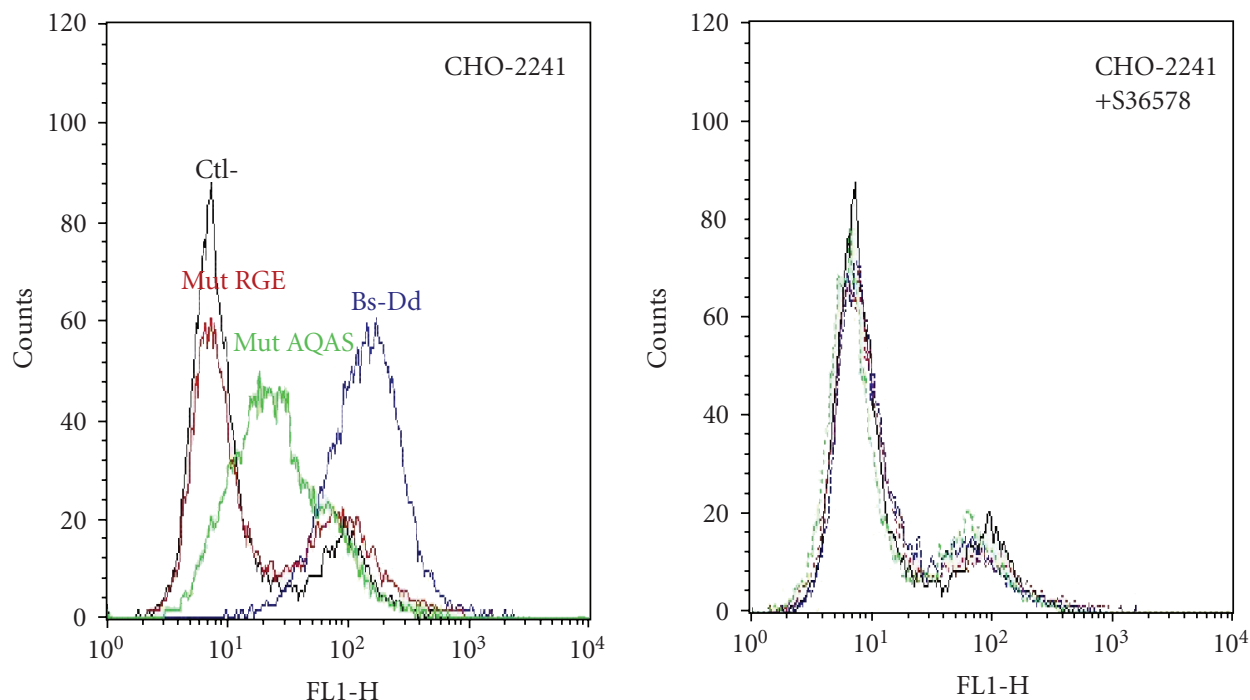

(b)

FIgure 3: FACS analysis of Bs-Dd and mutant binding at $4^{\circ} \mathrm{C}$ to HSPG expressing HeLa cells and HSPG deficient CHO-2241. (a) Particle binding to HeLa cells. Dodecahedra (wt or mutants) were incubated with HeLa cells in PBS $1 \mathrm{mM} \mathrm{CaCl}_{2}$ preincubated or not with S36578 drug (upper right and left panels, resp.). Lower panels show individual responses of the different mutants with or without S36578. (b) Particle binding to $\mathrm{CHO}-2241$ cells under the same conditions used above. Dodecahedra were allowed to bind to CHO-2241 cells preincubated or not with S36578 drug (right and left panels, resp.). Bound particles were detected by an antibody serum directed against the Ad3 penton base. 


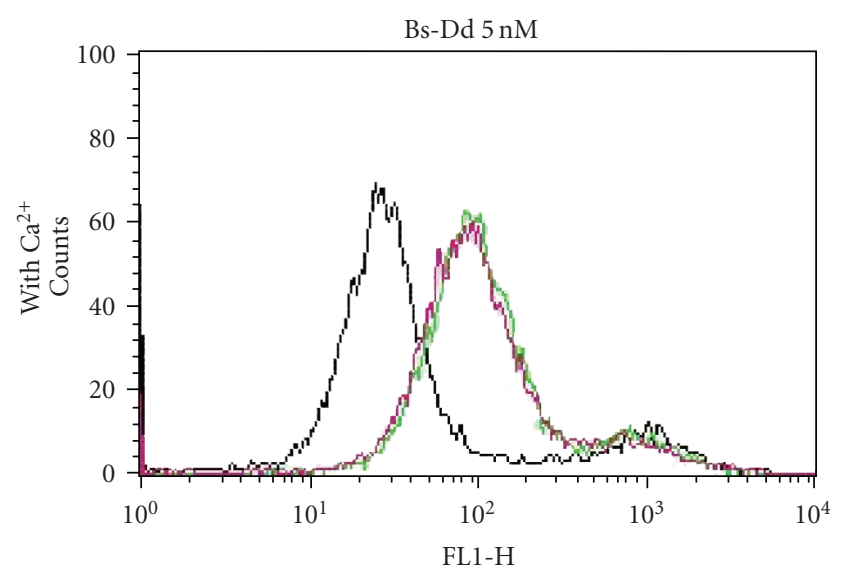

(a)

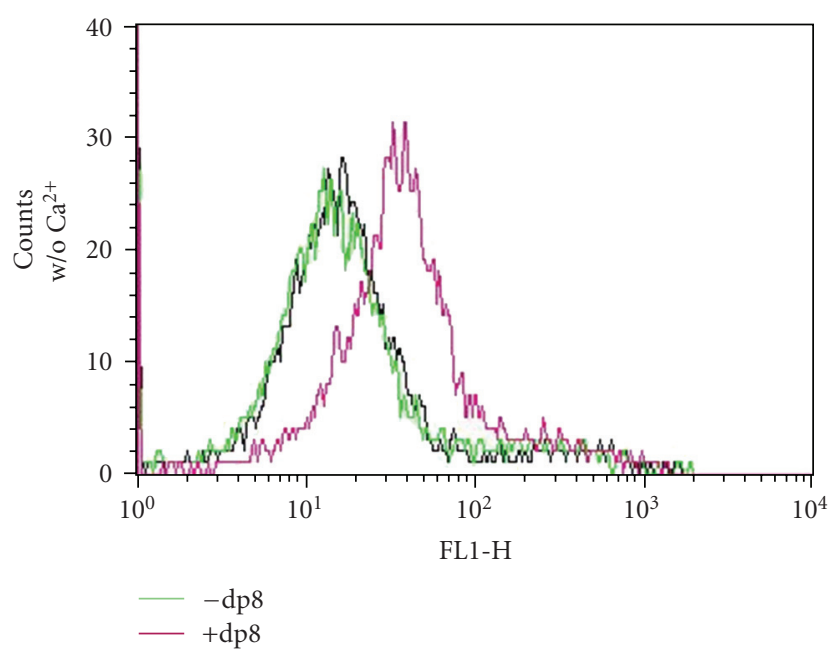

(c)

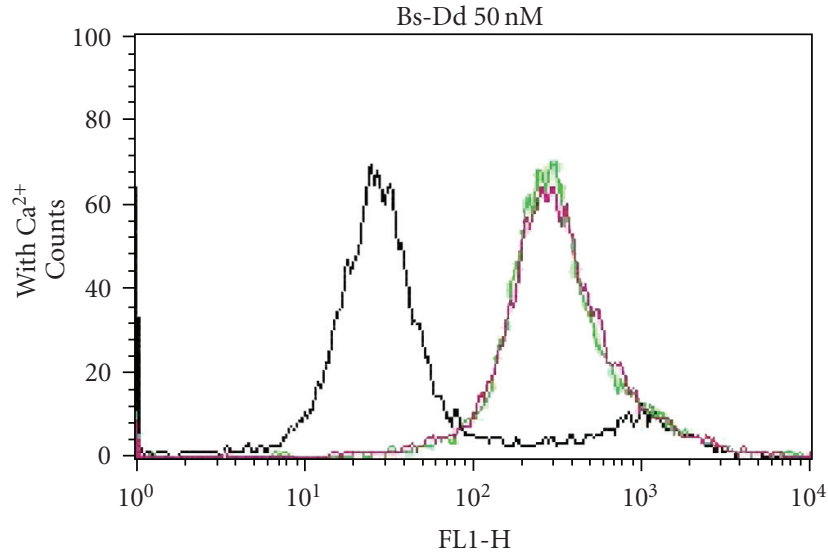

(b)

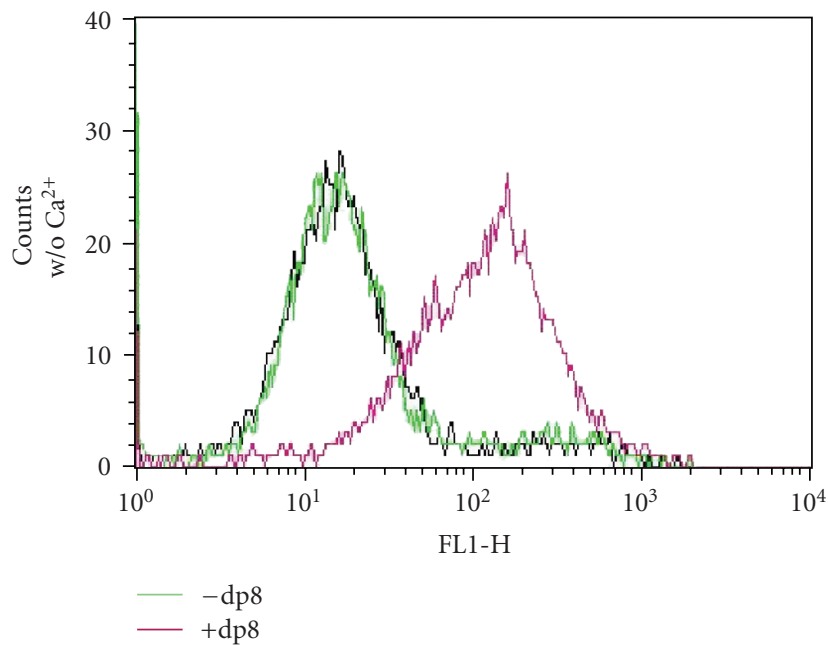

(d)

FIGURE 4: FACS analysis of the dp8 effect on Bs-Dd binding to HSPG deficient CHO-2241 cells in presence or absence of calcium. Bs-Dd was incubated at two different concentrations with $\mathrm{CHO}-2241$ cells at $4{ }^{\circ} \mathrm{C}$ in $\mathrm{PBS}$ supplemented or not with $1 \mathrm{mM} \mathrm{CaCl}_{2}$ (upper and lower panels, respectively). Bound Bs-Dd alone (green curves), or preincubated with dp8 oligosaccharide (purple curves), was detected by an antibody serum directed against the HAd3 penton base.

also for viruses [17]. Among them, Foot and Mouth Disease Virus (FMDV) and Adenovirus-Associated Virus (AAV-2) behave differently upon HSPG binding. Indeed, despite the close proximity of HSPG binding site located at the interface between VP1/VP2/VP3 proteins and the VP1 protein's RGD loop, no structural changes occur upon oligosaccharide binding on FMDV [18]. On the contrary, HSPG binding to the AAV-2 VP3 protein is thought to trigger structural changes on the adjacent integrin binding site and modulate its affinity according to a «click to fit» mechanism [19].

\section{Conclusion}

Our data supports the idea of a close relationship between HSPG and integrin receptors that might be finely tuned by structural changes upon docking to the cell surface. Our Cryo-EM reconstruction is an important step towards the understanding of this mechanism. Even though the biological role of dodecahedron is not yet understood, a better knowledge of the mechanism used by this particle to enter cells is of interest in biotechnology. Indeed, we have previously described the utility of dodecahedron in DNA and protein delivery $[3,6,7]$ and the comprehension of the entry mechanism used by this particle will be helpful in vectorology.

\section{Acknowledgments}

The authors are grateful to Dr. Gordon Tucker and the «Laboratoires Servier» for providing the S36578 drug and to Dr. Rabia Sadir for the dp8 oligosaccharide preparation. They thank Charles Vragniau for excellent technical assistance and Romain Vivès for corrections to the manuscript. This work is supported by the "Centre National de la Recherche Scientifique" (CNRS) and a grant from "Région Rhône-Alpes; Cluster 10 Infectiologie". 


\section{References}

[1] M.-T. Belin and P. Boulanger, "Involvement of cellular adhesion sequences in the attachment of adenovirus to the HeLa cell surface," Journal of General Virology, vol. 74, no. 8, pp. 1485-1497, 1993.

[2] T. J. Wickham, P. Mathias, D. A. Cheresh, and G. R. Nemerow, "Integrins alpha $\mathrm{v}$ beta 3 and alpha $\mathrm{v}$ beta 5 promote adenovirus internalization but not virus attachment," Cell, vol. 73, no. 2, pp. 309-319, 1993.

[3] P. Fender, R. W. H. Ruigrok, E. Gout, S. Buffet, and J. Chroboczek, "Adenovirus dodecahedron, a new vector for human gene transfer," Nature Biotechnology, vol. 15, no. 1, pp. 52-56, 1997.

[4] R. R. Vives, H. Lortat-Jacob, J. Chroboczek, and P. Fender, "Heparan sulfate proteoglycan mediates the selective attachment and internalization of serotype 3 human adenovirus dodecahedron," Virology, vol. 321, no. 2, pp. 332-340, 2004.

[5] P. Fender, G. Schoehn, F. Perron-Sierra, G. C. Tucker, and H. Lortat-Jacob, "Adenovirus dodecahedron cell attachment and entry are mediated by heparan sulfate and integrins and vary along the cell cycle," Virology, vol. 371, no. 1, pp. 155-164, 2008.

[6] P. Fender, G. Schoehn, J. Foucaud-Gamen, et al., "Adenovirus dodecahedron allows large multimeric protein transduction in human cells," Journal of Virology, vol. 77, no. 8, pp. 4960-4964, 2003.

[7] A. Garcel, E. Gout, J. Timmins, J. Chroboczek, and P. Fender, "Protein transduction into human cells by adenovirus dodecahedron using WW domains universal adaptors," Journal of Gene Medicine, vol. 8, no. 4, pp. 524-531, 2006.

[8] P. Fuschiotti, G. Schoehn, P. Fender, et al., "Structure of the dodecahedral penton particle from human adenovirus type 3," Journal of Molecular Biology, vol. 356, no. 2, pp. 510-520, 2006.

[9] G. Schoehn, P. Fender, J. Chroboczek, and E. A. Hewat, "Adenovirus 3 penton dodecahedron exhibits structural changes of the base on fibre binding," The EMBO Journal, vol. 15, no. 24, pp. 6841-6846, 1996.

[10] P. L. Stewart, C. Y. Chiu, S. Huang, et al., "Cryo-EM visualization of an exposed RGD epitope on adenovirus that escapes antibody neutralization," The EMBO Journal, vol. 16, no. 6, pp. 1189-1198, 1997.

[11] A. D. Cardin and H. J. R. Weintraub, "Molecular modeling of protein-glycosaminoglycan interactions," Arteriosclerosis, vol. 9, no. 1, pp. 21-32, 1989.

[12] T. J. Wickham, M. E. Carrion, and I. Kovesdi, "Targeting of adenovirus penton base to new receptors through replacement of its RGD motif with other receptor-specific peptide motifs," Gene Therapy, vol. 2, no. 10, pp. 750-756, 1995.

[13] C. F. Barbas III, L. R. Languino, and J. W. Smith, "Highaffinity self-reactive human antibodies by design and selection: targeting the integrin ligand binding site," Proceedings of the National Academy of Sciences of the United States of America, vol. 90, no. 21, pp. 10003-10007, 1993.

[14] C. M. S. Fabry, M. Rosa-Calatrava, J. F. Conway, et al., "A quasi-atomic model of human adenovirus type 5 capsid," The EMBO Journal, vol. 24, no. 9, pp. 1645-1654, 2005.

[15] J.-O. Lee, L. A. Bankston, M. A. Arnaout, and R. C. Liddington, "Two conformations of the integrin A-domain (Idomain): a pathway for activation?" Structure, vol. 3, no. 12, pp. 1333-1340, 1995.
[16] J.-P. Xiong, T. Stehle, R. Zhang, et al., "Crystal structure of the extracellular segment of integrin $\alpha \mathrm{V} \beta 3$ in complex with an Arg-Gly-Asp ligand," Science, vol. 296, no. 5565, pp. 151-155, 2002.

[17] S. Raman, T.-H. Hsu, S. L. Ashley, and K. R. Spindler, "Usage of integrin and heparan sulfate as receptors for mouse adenovirus type 1," Journal of Virology, vol. 83, no. 7, pp. 28312838, 2009.

[18] E. E. Fry, S. M. Lea, T. Jackson, et al., "The structure and function of a foot-and-mouth disease virus-oligosaccharide receptor complex," The EMBO Journal, vol. 18, no. 3, pp. 543554, 1999.

[19] A. Asokan, J. B. Hamra, L. Govindasamy, M. AgbandjeMcKenna, and R. J. Samulski, "Adeno-associated virus type 2 contains an integrin $\alpha 5 \beta 1$ binding domain essential for viral cell entry," Journal of Virology, vol. 80, no. 18, pp. 8961-8969, 2006. 

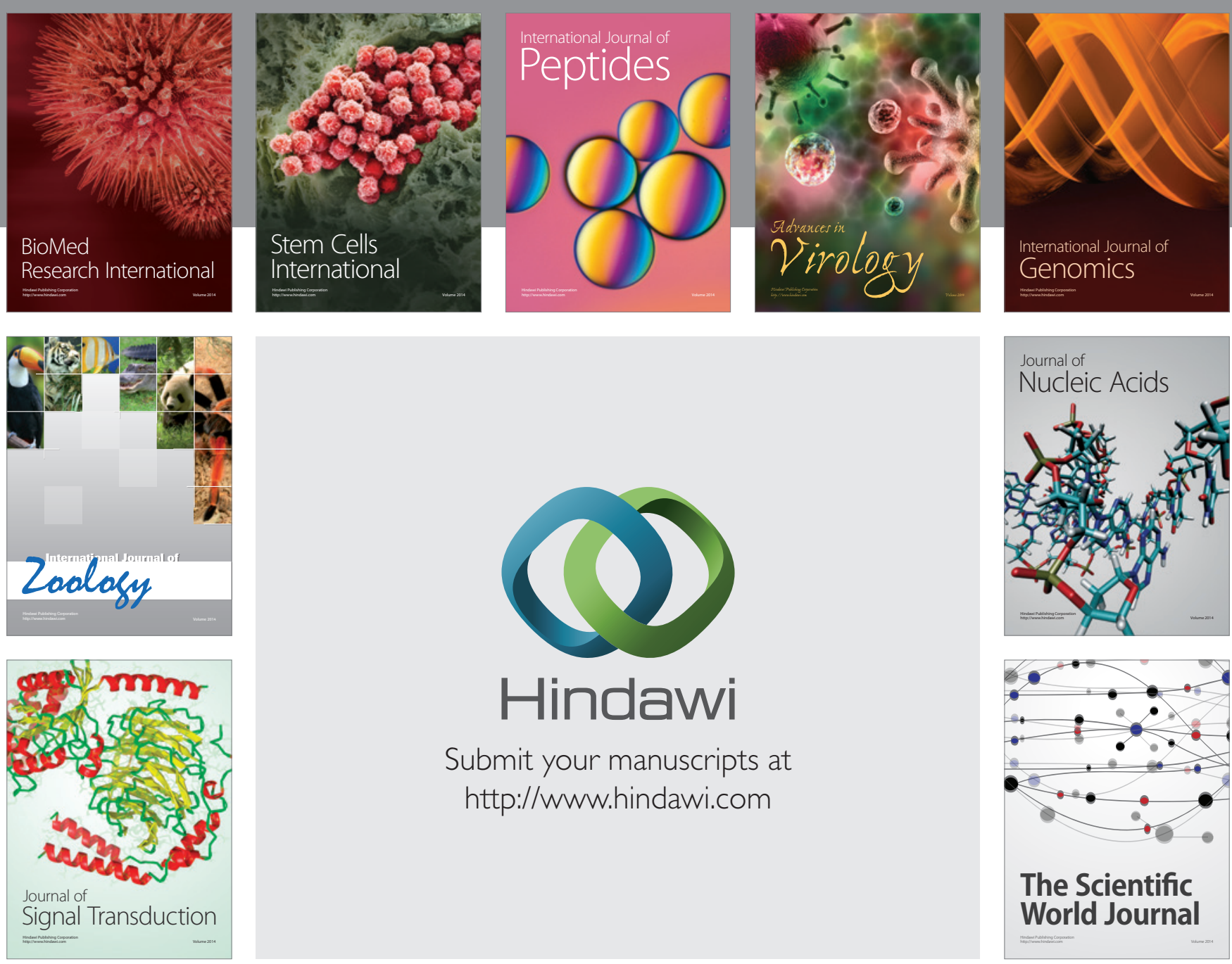

Submit your manuscripts at

http://www.hindawi.com
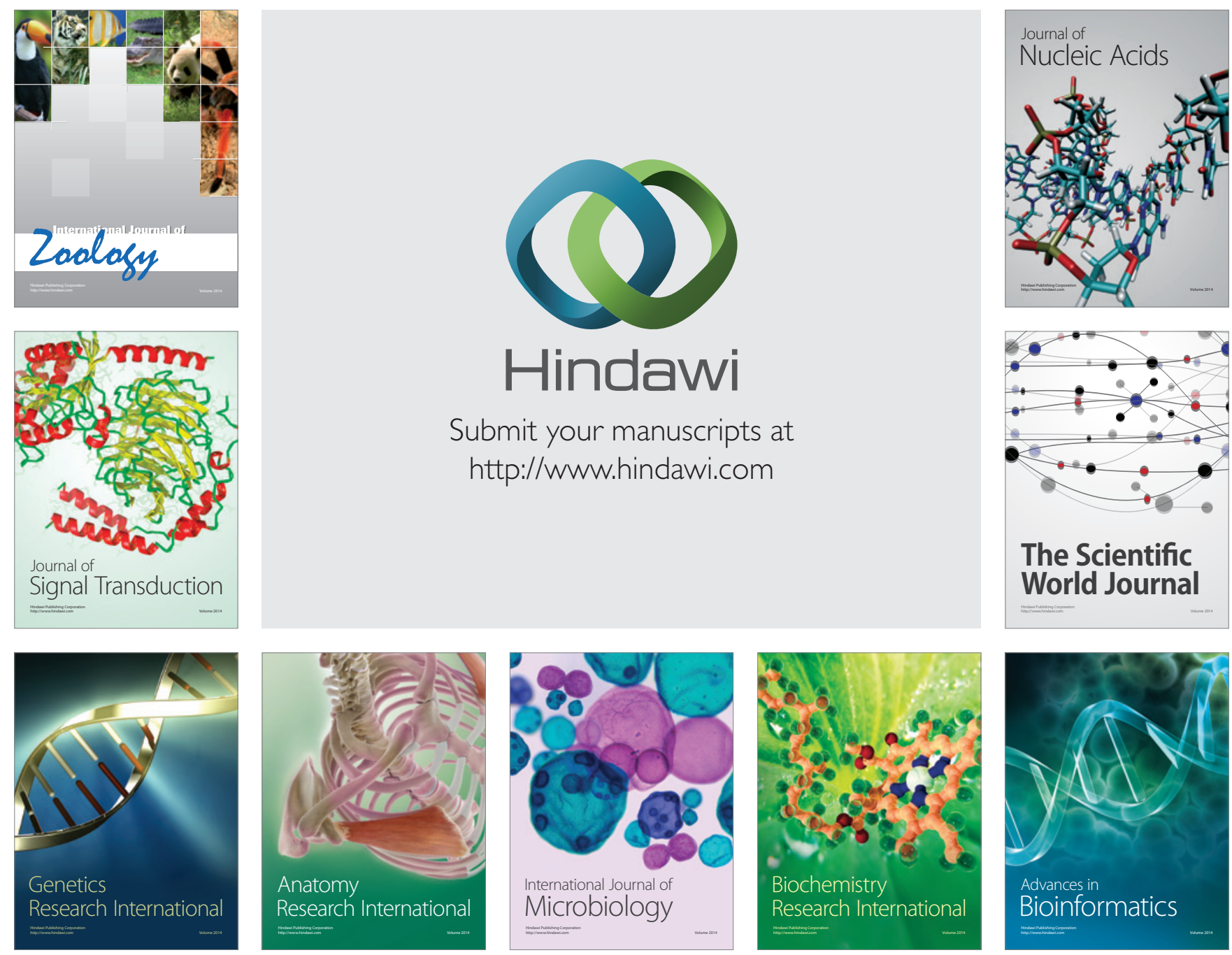

The Scientific World Journal
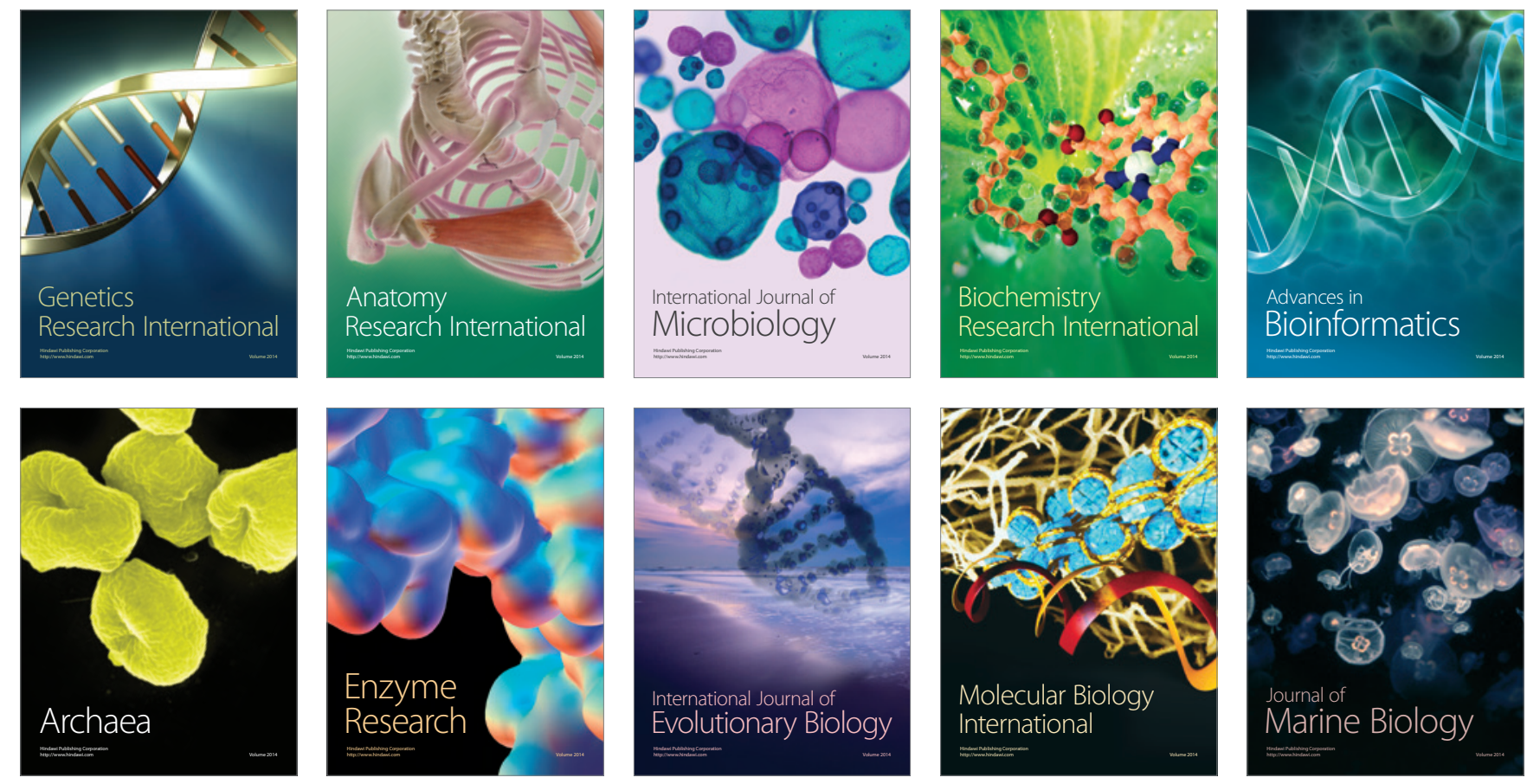